\title{
Magnetotransport as a diagnostic of spin reorientation: Kagome ferromagnet as a case study
}

\author{
Neeraj Kumar $\odot$ and Y. Soh $\oplus^{*}$ \\ Paul Scherrer Institute, 5232 Villigen, Switzerland \\ Yihao Wang $\odot$ and Y. Xiong \\ Anhui Province Key Laboratory of Condensed Matter Physics at Extreme Conditions, High Magnetic Field Laboratory \\ of the Chinese Academy of Sciences, Hefei 230031, China
}

(Received 23 August 2019; published 17 December 2019)

\begin{abstract}
We study the spin reorientation in $\mathrm{Fe}_{3} \mathrm{Sn}_{2}$ by examining the anisotropic magnetoresistivity in detail, exploiting the dependence of the resistivity on the direction between magnetization and applied current. We determine the distribution of the magnetic domains as a function of temperature between $360 \mathrm{~K}$ and $2 \mathrm{~K}$ and find the reorientation transition to peak at $120 \mathrm{~K}$. We discover that both out-of-plane and in-plane phases coexist at temperatures around the spin reorientation, indicative of a first-order phase transition. Although the volume of the magnetic domains in the different phases sharply changes at the spin reorientation transition, no appreciable changes in the electronic structure for a specific magnetization is detected by anisotropic magnetoresistivity at the spin reorientation. In contrast, we observe an electronic transition around $40 \mathrm{~K}$, hitherto unreported, and reflected in both the zero-field resistivity and anisotropic resistivity.
\end{abstract}

DOI: 10.1103/PhysRevB.100.214420

\section{INTRODUCTION}

While in most ferro or antiferromagnetic materials there is a unique crystallographic direction, including crystallographically equivalent directions, in which the moments like to point due to spin-orbit coupling, in some, the direction of the spin reorients as a function of a certain physical parameter such as temperature, pressure, etc. $\mathrm{Fe}_{3} \mathrm{Sn}_{2}$ is a kagome ferromagnet that undergoes a spin reorientation near $150 \mathrm{~K}$. While it has been reported that the moments in $\mathrm{Fe}_{3} \mathrm{Sn}_{2}$ point perpendicular to the kagome plane at high temperatures and parallel to the kagome plane at low temperatures, how the distribution of the magnetic domains in the two different spin orientations evolve throughout the spin reorientation is not well known.

Besides the unexplained magnetic transition, $\mathrm{Fe}_{3} \mathrm{Sn}_{2}$ has recently attracted much attention due to the layered kagome lattices on which the $\mathrm{Fe}$ atoms reside; the latter were suggested as leading to flat bands which could host the fractional quantum Hall effect at room temperature without an external magnetic field [1]. There have since then been claims of observations of such flat bands [2], as well as of Dirac-like cones centered at energies well below $(0.1 \mathrm{eV})$ the Fermi surface [3], Weyl nodes at the Fermi level [4], and "giant" spin-orbit tunability [5], where the magnetization has strong influence on the electronic properties due to the combination of strong spin-orbit coupling and nontrivial band structure. Therefore, a precise understanding of the spin reorientation is necessary to understand the nontrivial electronic properties of this system.

\footnotetext{
*Corresponding author: yona.soh@psi.ch
}

Kagome $\mathrm{Fe}_{3} \mathrm{Sn}_{2}$ orders ferromagnetically below a Curie temperature of $T_{C} \approx 640 \mathrm{~K}$ based on SQUID magnetometry [6]. Previous studies using Mossbauer spectroscopy reported a Curie temperature of $612 \mathrm{~K}$ [7] and $657 \mathrm{~K}$ [8]. Below the ordering temperature, the easy axis of magnetization is parallel to the crystallographic $c$ axis. Initial study using Mossbauer spectroscopy noticed that a spin reorientation transition (SRT) occurs at $114 \mathrm{~K}$ [7]. Following studies using Mossbauer spectroscopy suggested that below $220 \mathrm{~K}$, there are abrupt spin rotations occurring over a large temperature range 0 $220 \mathrm{~K}$ with the spin direction close to the $a b$ kagome plane at low temperatures [8]. Further studies investigating the spin rotation using neutron diffraction combined with Mossbauer spectroscopy noted that the rotation is more complicated than a continuous rotation described by a unique angle or a simple abrupt rotation [9].

The SRT was recently revisited, using powder neutron diffraction, where the transition was suggested to occur over a large temperature range from 570 to $75 \mathrm{~K}$ [6]. The order of the transition is not discussed in any of the previous reports and they report very broad transitions. Furthermore, previous reports measured powder sample, where the outcome could be representative of nonintrinsic features of the material. In the case of $\mathrm{DyFe}_{11} \mathrm{Ti}$, it has been very clearly shown that the sample quality can easily affect the sharpness of the SRT [10]. In this work, we investigate the magnetic behavior especially the SRT using high-quality single crystals of $\mathrm{Fe}_{3} \mathrm{Sn}_{2}$. We use magnetotransport measurements as the main probe, and focus on the anisotropy in the resistivity due to the angle between magnetization and applied current. Anisotropic magnetoresistance (AMR) is a very useful tool to probe the bulk domain configuration, especially if the magnetoresistance (MR) is not very high [11]. Although recently AMR was used to infer the 

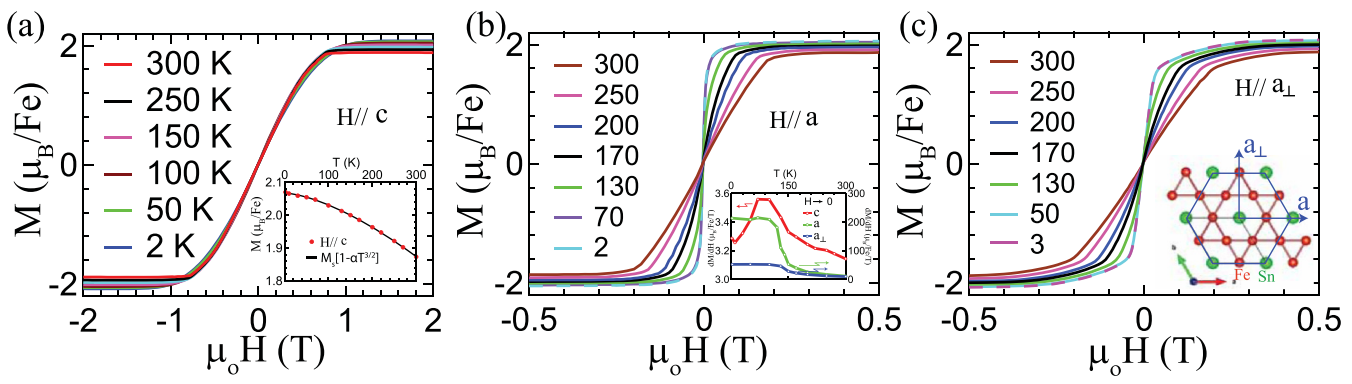

FIG. 1. Magnetization ( $M$ ) for magnetic field applied along (a) the $c$ axis, (b) the $a$ axis, and (c) the $a_{\perp}$ axis at various temperatures. Inset (a) Saturation magnetization along the $c$ axis as a function of temperature. Inset (b) Susceptibility for magnetic field along each of the three directions in the limit of zero field. Inset (c) Kagome plane together with the directions along which field has been applied in the plane.

existence of SRT in ruthenates, the study mainly relied on the change of the sign of the low-field MR at the SRT and did not exploit the AMR in a quantitative way as we do in our current work [12].

While there have been several reports on the large anomalous Hall effect in $\mathrm{Fe}_{3} \mathrm{Sn}_{2}[13,14]$, MR in $\mathrm{Fe}_{3} \mathrm{Sn}_{2}$ has not been investigated in detail so far, except for MR for $H \| \mathbf{c}[3,14]$. We investigate in detail the temperature dependence of MR for fields along different directions and discuss the various contributions. Using AMR, we examine the SRT in detail and unambiguously demonstrate the first-order nature of the transition. We find the transition range to be much narrower $70-150 \mathrm{~K}$ than previous reports, with the transition peaked at $120 \mathrm{~K}$. We speculate the reason for the broad transition observed in previous studies to be due to the samples being in powder form. Our magnetoresistivity data is compared against bulk magnetization measurements to understand the influence of the magnetization state on the resistance.

In addition to the SRT, we discovered that there is an electronic transition at $T=40 \mathrm{~K}$ based on AMR and zero field resistivity data. The origin of this transition is not clear to us at present.

\section{EXPERIMENTAL DETAILS}

The bottom inset in Fig. 2(a) shows the crystal structure of $\mathrm{Fe}_{3} \mathrm{Sn}_{2}$ with the kagome plane shown in Fig. 1(c) inset. Hexagonal bilayers of $\mathrm{Fe}_{3} \mathrm{Sn}$ in the $a b$ plane are stacked along the $c$ axis, separated by Sn hexagonal layers [15]. Crystals were grown using vapor transport. As-grown crystals are in the form of thin platelets and carry the hexagonal crystal structure morphology. Structure of the crystal is confirmed using single crystal XRD and Laue diffraction. A crystal of lateral dimensions $1 \times 1 \mathrm{~mm}^{2}$ and thickness of $35 \mu \mathrm{m}$ was selected for the measurements. Magnetization measurements were carried out using a Quantum Design SQUID VSM. Magnetotransport measurements were conducted using a Quantum Design PPMS with the option to rotate the sample with respect to the magnetic field in-situ. The long side of the crystal was found to be parallel to the crystallographic $a$ axis and this direction was selected for applying current. Magnetotransport measurement was performed in three configurations: (i) parallel configuration, i.e., magnetic field was applied parallel to the current along a, (ii) transverse configuration, with magnetic field in the kagome plane but perpendicular to the current; we denote this direction as $\mathbf{a}_{\perp}$, and (iii) out-of-plane c configuration. Magnetization was also measured for these directions. The in-plane directions are shown in Fig. 1(c) inset.

\section{RESULTS AND DISCUSSION}

We examine the bulk magnetometry as shown in Fig. 1. As $\mathrm{Fe}_{3} \mathrm{Sn}_{2}$ is a soft ferromagnet, no hysteresis is observed in the magnetization. For $H \| \mathbf{a}$, a clear change in saturation field and slope of $M$ versus $H$ curve is seen upon lowering the temperature, confirming the SRT. Similar behavior for $H \| \mathbf{a}_{\perp}$ is also seen which shows an additional turn in $M$ versus $H$ curve and higher saturation field. A higher saturation field could be either due to anisotropy energy or due to the demagnetization factor because the sample geometry is elongated along a, or both. As seen in Fig. 1(a), for $H \| \mathbf{c}$, change in the $M$ versus
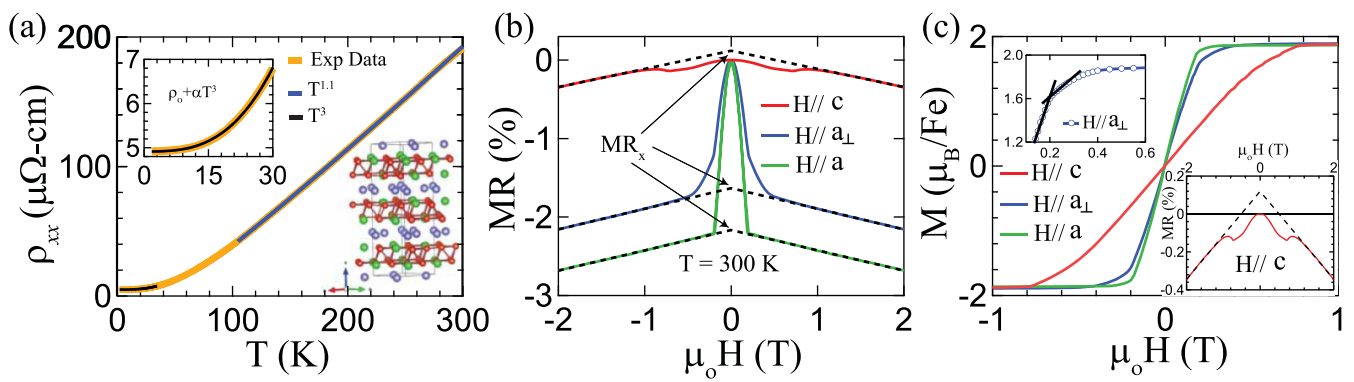

FIG. 2. (a) Resistivity vs. temperature showing a typical metallic behavior with different fittings. Inset shows the crystal structure of Fe $\mathrm{Sn}_{2}$ showing stacking of kagome bilayers along the $c$ axis. Upper inset highlights the fitting below $30 \mathrm{~K}$. (b) Magnetoresistance (MR) for field along $\boldsymbol{a}, \boldsymbol{a}_{\perp}$, and $\boldsymbol{c}$ directions. (c) Magnetization $(M)$ for these directions. Top inset shows the turn in the magnetization for $H \| \mathbf{a}_{\perp}$. Bottom inset highlights the magnetoresistance (MR) for $H \| \mathbf{c}$. 

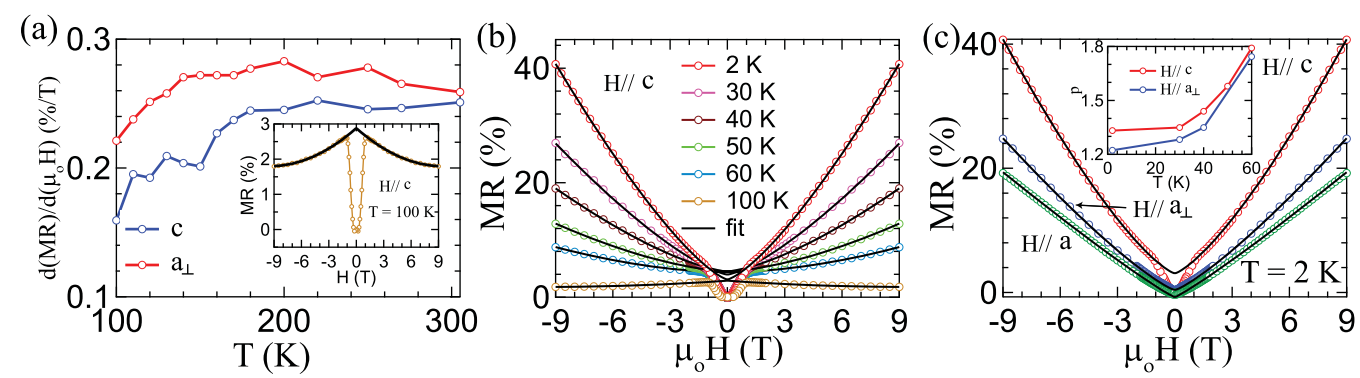

FIG. 3. (a) Electron-magnon scattering term as a function of temperature. Inset: Magnetoresistance (MR) for $H \| \mathbf{c}$ at $100 \mathrm{~K}$. (b) Magnetoresistance (MR) at various temperatures below $100 \mathrm{~K}$ for $H \| \mathbf{c}$, showing excellent fitting with a power law $\rho=\rho_{o}\left(1+\alpha H^{p}\right)$, except at $100 \mathrm{~K}$. (c) Magnetoresistance (MR) at $2 \mathrm{~K}$ for field along each of the three directions. Inset shows the change in exponent $p$ of the power law fitting.

$H$ curves depending on temperature is very small, which is due to the masking of changes in the magnetic anisotropy by the much larger demagnetization field. The inset in Fig. 1(b) shows the susceptibility $d M / d H$ at zero magnetic field as a function of temperature for a magnetic field applied along each of the three directions as derived from the $M$ versus $H$ data in Fig. 1. A clear change in the susceptibility along $\mathbf{a}$ and $\mathbf{a}_{\perp}$ beginning at $150 \mathrm{~K}$ on cooling, support the SRT. Based on the susceptibility data, we estimate that the SRT is completed at a temperature above $90 \mathrm{~K}$. For $H \| \mathbf{c}$, change in the susceptibility is very small, primarily again due to the demagnetization field. Figure 1(a) inset shows the saturation magnetization as a function of temperature for $H \| \mathbf{c}$. The magnetization follows Bloch's law $M=M_{S}\left(1-\alpha T^{\frac{3}{2}}\right)$.

The resistivity of $\mathrm{Fe}_{3} \mathrm{Sn}_{2}$ shows a typical metallic behavior with a very high residual resistivity ratio (defined as $\frac{\rho(300 \mathrm{~K})}{\rho(2 \mathrm{~K})}$ ) of 40 [Fig. 2(a)]. The resistivity value at room temperature is around $200 \mu \Omega-\mathrm{cm}$ and agrees well with other recent reports $[3,14]$. The resistivity is almost linear above $100 \mathrm{~K}$ but can be more appropriately described by a $T^{1.2}$ dependence. At low temperature below $30 \mathrm{~K}$, the resistivity varies as $T^{3}$. A $T^{3}$ dependence could indicate $s-d$ electron scattering as the possible predominant mechanism at low temperature [16]. As we will describe later, it is possible that a phase transition occurs around $30 \mathrm{~K}$.

Figure 2(b) shows the MR (defined as $\frac{\rho(H)-\rho(0)}{\rho(0)}$ ) of $\mathrm{Fe}_{3} \mathrm{Sn}_{2}$ at $300 \mathrm{~K}$ for a magnetic field applied in the $a b$ plane along longitudinal and transverse directions, and along out-of-plane direction ( $c$ axis). For each configuration, a negative MR is observed, which is linear above a certain saturation field. The saturation field for each direction reflected in the MR is in accordance with the corresponding saturation field in the magnetization as shown in Fig. 2(c). Correspondingly, this means that the MR features below the saturation field reflect changes in the magnetic domain configuration on application of magnetic field. We first focus our attention on the MR at fields above the saturation field in magnetization. It is to be noted that at low temperature, the MR becomes ultimately positive at high fields in contrast to the behavior at $300 \mathrm{~K}$. This positive MR is not associated with the magnetization and magnetic domains and is shown in Fig. 3 (see Fig. S1 in the Supplemental Material [17]).

The isotropic magnon scattering contribution persists down to nearly $150 \mathrm{~K}$ as shown in Fig. 3(a) as a negative slope of the MR above the saturation field. This contribution starts to decrease below $150 \mathrm{~K}$ as magnons freeze. The Lorentz MR is normally negligible at high temperature and starts to become significant only at low temperatures around $100 \mathrm{~K}$. The MR behavior at $100 \mathrm{~K}$ [Fig. 3(a) inset] shows a clear competition between the two effects and can be easily fitted to a polynomial $\rho(H)=\alpha H+\beta H^{2}$. A similar behavior has been seen in other ferromagnetic materials as well [18]. As the temperature decreases, the linear negative MR is no longer significant. Below $60 \mathrm{~K}$, the MR is completely positive and better described by a power law $\rho(H)=\rho_{o}\left(1+\alpha H^{p}\right)$ [Fig. 3(b)]. The exponent of the field is close to 1.8 at $60 \mathrm{~K}$ and to 1.3 at $2 \mathrm{~K}$. As noted in Ref. [19], various semi metallic materials are seen to exhibit positive MR with varying exponents. The polynomial $\rho(H)=\alpha H+\beta H^{2}$ also can be used to fit the MR, but the power law fitting is relatively better (see the Supplemental Material [17]). At $2 \mathrm{~K}$, positive MR is seen for field along the three directions studied in this paper [Fig. 3(c)], however, with different exponents. For current and field parallel to each other, the MR at $2 \mathrm{~K}$ is almost linear. Causes for the subquadratic MR has been ascribed to either the dependence of the mobility on the magnetic field [20] or due to the itinerant carriers encountering sharp cornered surfaces [21].

We now turn our attention to the low-field MR. The resistivity of a fully magnetized system in the limit of zero external field $\rho_{x}$, where $x$ refers to the direction of the magnetization, or the corresponding magnetoresistance $M R_{x}=$ $\left[\rho_{x}-\rho(0)\right] / \rho(0)$ is obtained by extrapolating the data at high field to zero field as shown in Fig. 2(b). $M R_{x}$ or $\rho_{x}$ reflects the anisotropic resistivity, which depends on the direction of the magnetization and arises from spin-orbit coupling. A positive value for $M R_{c}$ and a negative one for $M R_{a}$ and $M R_{a_{\perp}}$ is obtained due to $\rho_{c}>\rho_{a \perp}>\rho_{a}$ in our system. The zerofield resistivity $\rho(0)$ is determined by the magnetic domain distribution and associated anisotropic resistivities $\rho_{x}$ and its value lies in between $\rho_{a}$ and $\rho_{c}$. As the current is applied along a, we define the anisotropic magnetoresistance (AMR) ratio as $M R_{a}-M R_{c}$. An AMR ratio of $-2.2 \%$ is observed. A negative AMR ratio is opposite to the conventional behavior of ferromagnets [22]. However, it is seen in systems such as $\mathrm{Fe}_{3} \mathrm{O}_{4}$ and 2D ferromagnets such as $\mathrm{Cr}_{2} \mathrm{Ge}_{2} \mathrm{Te}_{6}$ and $\mathrm{Fe}_{3} \mathrm{GeTe}_{2}$ [23]. Conduction by the minority spin carriers has been suggested as the reason behind a negative AMR ratio [22]. 
The magnitude of $M R_{c}$ at $300 \mathrm{~K}$ is much smaller than the corresponding values for the other magnetization directions, which is in line with $\mathbf{c}$ being the easy axis at room temperature. However, for all the magnetic domains to be along $\mathbf{c}$, $M R_{c}$ should have been zero, as both parallel and antiparallel domains contribute equally to the resistivity. A nonzero $M R_{c}$ means that (i) either the magnetization axis is oriented close to but not exactly parallel to the $c$ axis or (ii) the sample consists of domains with multiple orientations with domains along $\mathbf{c}$ being a significantly larger fraction. As we will show later, the latter is more likely to be the case. We estimate the out-of-plane domain fraction using the zero field anisotropic resistivity values $\rho_{x}$. We assume an effective medium model

$$
(1-x) \rho_{c}+\frac{x\left(\rho_{a}+\rho_{a_{\perp}}\right)}{2}=\rho(0),
$$

where $x$ is the volume fraction of in-plane magnetic domains and the two in-plane directions are averaged to represent the in-plane resistivity. At $300 \mathrm{~K}, x$ is found to be 0.08 . We apply this concept later to extract the temperature dependence of the distribution of magnetic domains and show that the assumption of having a volume distribution of magnetization with different magnetic anisotropy is justified based on our MR data.

For $H \| \mathbf{a}$, and $H \| \mathbf{a}_{\perp}$, the large negative MR at $300 \mathrm{~K}$ below the saturation field is due to the rotation of the magnetization from the high resistivity $M \| \mathbf{c}$ state to the low resistivity $M \| a b$ plane configuration. For $H \| \mathbf{a}_{\perp}$, however, there is an extra change in slope before saturation and an overall higher saturation field. This is because $\mathbf{a}_{\perp}$ is not a high symmetry axis. When the field is applied along $\mathbf{a}_{\perp}$, the magnetic domains first align toward a high symmetry axis, and later eventually toward $\mathbf{a}_{\perp}$ as the field becomes stronger. This behavior near saturation is also seen in the magnetization data as shown in Fig. 1(c).

As shown in the bottom inset of Fig. 2(c), for $H \| \mathbf{c}$, the $\mathrm{MR}$ is nonmonotonic below the saturation field. Up to $0.7 \mathrm{~T}$, the MR decreases with the increasing field and then after that increases rapidly up to $1 \mathrm{~T}$. Such behavior can be explained by the rotation of in-plane domains on application of a field in the presence of a tilted secondary easy axis at a small angle with respect to the $a b$ plane. The in-plane fraction of domains will rotate toward this easy axis at low field if the anisotropy energy is lower as compared to the anisotropy energy for the out-of-plane rotation. At higher field, these domains will eventually rotate toward the $c$ axis causing an increase in the MR [24]. For comparatively lower resistivity for the secondary axis direction with respect to the initial state, which is possible due to in-plane anisotropy as seen in Fig. 2(b), a minimum in resistivity for $H \| \mathbf{c}$ will occur.

Similar MR behavior for $\mathrm{Fe}_{3} \mathrm{Sn}_{2}$ was seen previously [25], where the field values at the lowest MR and the highest MR were explained as the transition points from bubble to skyrmionic bubble and further to ferromagnetism phase, respectively. While it could be possible that there is an accompanied change of the magnetic phase, we can explain the trend in the MR based on the magnetic domain behavior as explained above without invoking skyrmionic bubbles. Further, we believe that this small volume of in-plane domains arises from a competition between in-plane and out-of-plane mag- netic anisotropy, which facilitates the formation of skyrmionic bubbles. Above the saturation field, the MR is linear in all the cases and is caused by the suppression of electron-magnon scattering on application of a magnetic field as discussed previously [18].

To further understand the magnetic domain composition, we explore the temperature dependence of the low-field MR. Figures 4(a) and 4(b) show the MR for $H \| \mathbf{a}$ at various temperatures between $360 \mathrm{~K}$ and $2 \mathrm{~K}$. As seen in Fig. 4(a), as the temperature decreases from 360 to $140 \mathrm{~K}$, the negative $M R_{a}$ increases in magnitude from $2 \%$ at $360 \mathrm{~K}$ up to $4 \%$ at $140 \mathrm{~K}$. Below $140 \mathrm{~K}$, there is a decrease in the magnitude of $M R_{a}$ with decreasing temperature. $M R_{a}$ decreases to $0.3 \%$ at $2 \mathrm{~K}$. The saturation field monotonically decreases with the decreasing temperature in accordance to the magnetization data.

Figures 4(c) and 4(d) show the corresponding data for $H \| \mathbf{a}_{\perp}$. Similar to the above, the magnitude of the negative $\mathrm{MR}$ increases with decreasing temperature up to $150 \mathrm{~K}$ and decreases after that. However, in this case, $M R_{a_{\perp}}$ becomes positive below $80 \mathrm{~K}$. For $H \| \mathbf{c}$, where $M R_{c}$ is positive at $300 \mathrm{~K}$, the positive $M R_{c}$ becomes more positive with decreasing temperature. These observations are compiled in Fig. 5(a), which shows the temperature dependence of $M R_{x}$ between 2 and $300 \mathrm{~K}$. While in most of the cases, $M R_{x}$ is estimated by a linear extrapolation of the data above saturation as shown in Fig. 2(b), at low temperatures a power law is used to extrapolate the positive MR to zero field.

As shown in Fig. 5(a), at $150 \mathrm{~K}$, the trend of $M R_{x}$ changes for all three directions, suggesting the spin reorientation transition as the common origin, where the majority of the domains reorient from the $c$ axis into the $a b$ plane. Using the same effective medium model, as shown in Eq. (1), the volume fraction of the in-plane magnetic domains is calculated and shown in Fig. 5(b) at temperatures ranging from $305 \mathrm{~K}$ down to $2 \mathrm{~K}$. The volume fraction curve clearly reveals the SRT. From $300 \mathrm{~K}$ toward $150 \mathrm{~K}$, there is a slow increase in in-plane volume fraction, followed by a rapid increase below $150 \mathrm{~K}$. At $80 \mathrm{~K}, 90 \%$ volume fraction is magnetized in the $a b$ plane. At $70 \mathrm{~K}$, roughly the entire sample is magnetized in the $a b$ plane. By taking a derivate of the curve, the SRT peaks around $120 \mathrm{~K}$. It is to be noted that an assumption regarding the equal population of domains in $\mathbf{a}$ and $\mathbf{a}_{\perp}$ direction is made, which likely is not strictly true since $\mathbf{a}$ is a principal axis but $\mathbf{a}_{\perp}$ is not. Nevertheless, it is sufficient to provide a good estimate of the transition behavior.

We can qualitatively explain the temperature dependence of the low-field MR and $M R_{x}$ based on the SRT and volume fraction of magnetic domains. From $300 \mathrm{~K}$ down to $150 \mathrm{~K}$, the majority of the domains are magnetized parallel to the $c$ axis. Therefore, in the parallel and transverse configuration, the application of a magnetic field will force these domains to rotate its magnetization to the $a b$ plane leading to a lower resistivity and correspondingly to a negative $M R_{x}$, whereas there would be little $M R_{x}$ for the out-of-plane configuration. However, as the volume fraction of in-plane domains start increasing below $150 \mathrm{~K}$, the application of a magnetic field in the parallel and transverse configuration would have a smaller effect than before, resulting in the decrease of the magnitude of the negative $M R_{x}$ causing the reversal of the trend at $150 \mathrm{~K}$. For the magnetic field along out-of-plane direction, 

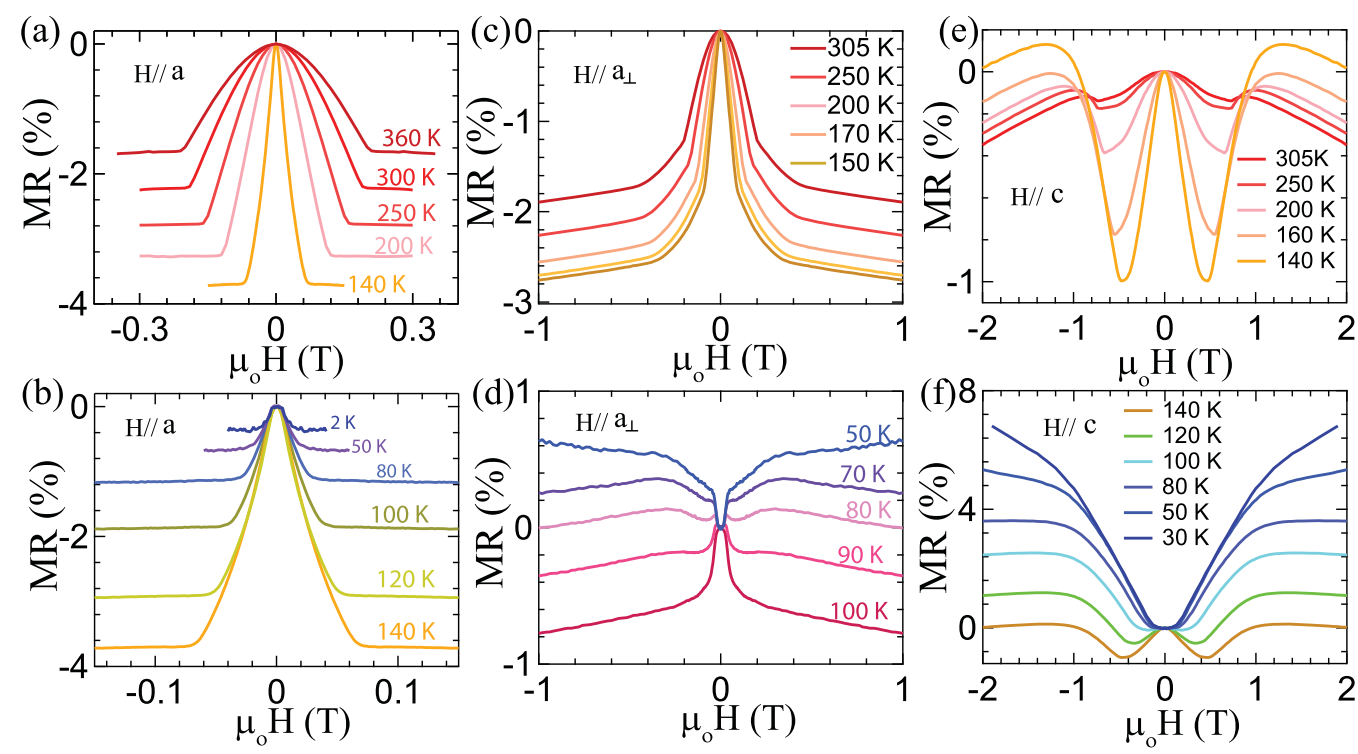

FIG. 4. Magnetoresistance at several temperatures for magnetic field along each of the three directions (a, b) the $a$ axis, (c, d) the $a_{\perp}$ axis, and (e, f) the $c$ axis.

the increase of in-plane domains below $150 \mathrm{~K}$ will, however, increase the positive $M R_{c}$ as the increased in-plane domains with lower anisotropic resistivity will have to rotate into the out-of-plane direction with higher anisotropic resistivity. The sign change from negative to positive $M R_{a_{\perp}}$ around $80 \mathrm{~K}$ in the transverse configuration can be understood based on the completion of the SRT with the magnetic easy axis being along directions equivalent to a. Above $80 \mathrm{~K}$, there is still some volume fraction with magnetic domains along $\mathbf{c}$. Therefore, the MR and $M R_{a_{\perp}}$ is negative. However, once the SRT is complete with the magnetic domains being magnetized along directions equivalent to a, the zero-field resistivity is lower than the anisotropic resistivity in the transverse configuration, giving rise to a positive MR and $M R_{a_{\perp}}$. Recently, the low field negative MR for $H$ in the $a b$ plane at $200 \mathrm{~K}$ has been attributed to weak localization [26]. However, we find $200 \mathrm{~K}$ too high to observe weak localization and the temperature dependence of the magnitude of the negative MR below $150 \mathrm{~K}$, which shows a decrease on cooling, is inconsistent with a weak localization picture.

Coming back to the butterfly MR for $H \| \mathbf{c}$, the magnitude of the negative MR reflected in the minima increases with decreasing temperature up to $150 \mathrm{~K}$, which is consistent with slowly increasing $a b$-plane domain volume. The decrease in magnitude of this MR minimum below $150 \mathrm{~K}$ is again consistent with the SRT, where, although the $a b$-plane domain volume increases, the MR associated with $a b$ domains decreases rapidly.

Comparing the data in Figs. 4(a) and 4(b), we observe a qualitative change in the shape of the MR versus $H$ curve from a dome to a tower shape at low temperature. We argue that this change in shape is associated with the different magnetic domain configuration. The derivative of the MR, as shown in Fig. 5(c) further emphasizes this point, where at high temperatures the derivative peaks at a higher field and at low temperatures at a lower field. This difference is due to the difference in the anisotropy energy of c domains, which are majority domains at high temperature, and $a b$-plane domains not parallel to $\mathbf{a}$, which are the cause of MR and $M R_{a}$ at low temperature. The derivative curves for the intermediate temperature range such as at 150 and $200 \mathrm{~K}$, reflect a superposition of two peak shapes described above. This shows that the SRT does not occur by a continuous gradual rotation of $M$ in the whole sample as in a second-order phase transition but rather through a co-existence of both out-ofplane and in-plane domains whose volume fraction changes
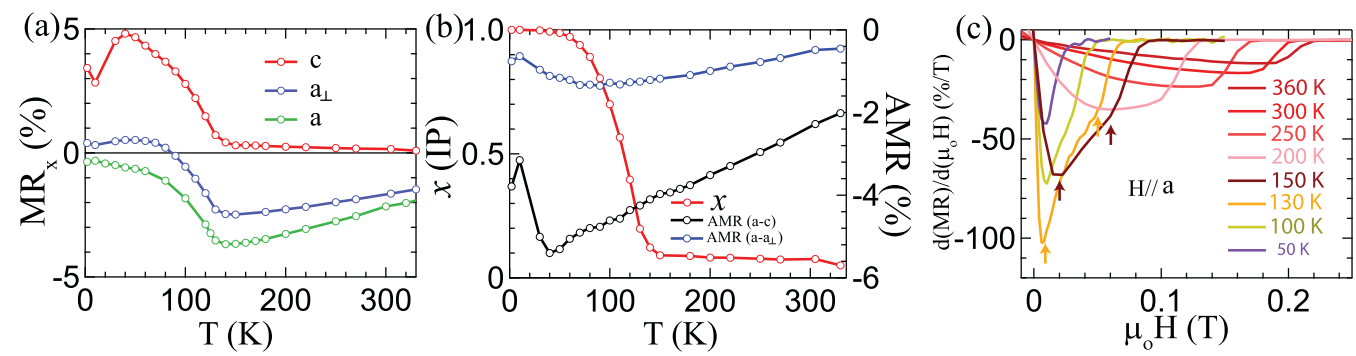

FIG. 5. (a) Evolution of $M R_{x}$ with temperature for the three magnetization directions. (b) Volume fraction of in-plane domains $x$ is shown on the left axis. The right axis shows the AMR ratio for two different configurations. (c) First derivate of magnetoresistance for $H \| \mathbf{a}$ clearly highlights the change in the easy axis and coexistence of two different phases at some of the temperatures. 
as the SRT occurs. The in-plane domain volume increases as the out-of-plane domain volume decreases as the system is cooled. This coexistence is the hallmark of a first-order phase transition and validates our assumption of associating a volume fraction and AMR for each magnetization direction to describe the measured resistivity at a given temperature.

We now turn our attention to the magnitude of the AMR ratio. As seen in Fig. 5(b), the AMR ratio does not show any visible change at the SRT, which suggests that the electronic structure corresponding to each magnetization direction probed by AMR does not change at the SRT even though the volume fraction of different magnetization directions undergo a rapid redistribution at the SRT. Rather, at low temperature around $40 \mathrm{~K}$, we observe a decrease in the magnitude of the AMR ratio between $\rho_{c}$ and $\rho_{a}$ which seems to be mainly driven by the decrease in $\rho_{c}$. The reason behind this decrease is not clear. The zero-field resistivity also displayed a unique temperature dependence below $40 \mathrm{~K}$. We speculate that there is an electronic transition around $40 \mathrm{~K}$, with the transitions observed in AMR and zero-field resistivity having the same origin. For the AMR ratio between $\mathbf{a}$ and $\mathbf{a}_{\perp}$, a similar trend is seen, however their extremum is seen around $80 \mathrm{~K}$. Further experiments will be needed to discern the source of these anomalies in the data.

\section{CONCLUSION}

We have been able to gain insight about the spin reorientation in $\mathrm{Fe}_{3} \mathrm{Sn}_{2}$ such as the distribution of the magnetic domains in the high- versus low-temperature phase, the transition temperature as the system undergoes a spin reorientation, and evidence for phase coexistence by probing the magnetoresistance. Our results rule out the scenario of a continuous rotation of the magnetic easy axis and are consistent with a report describing the first-order nature of the spin reorientation transition in $\mathrm{Fe}_{3} \mathrm{Sn}_{2}$ probed by SQUID magnetometry and MFM [27].

In the studies performed with SQUID magnetometry, jumps in the magnetization were observed on cooling around $130 \mathrm{~K}$ and at slightly higher temperatures depending on the strength of the external magnetic field applied in the $a b$ plane, pointing to a first-order phase transition with the transition temperature being consistent with the temperature of $120 \mathrm{~K}$ at which the SRT peaks in our MR studies. The difference in the peak of the transition of about $10 \mathrm{~K}$ between the two studies could be attributed to the strength of the applied magnetic field and sample geometry, which affect the transition temperature due to the dipolar interactions. A larger magnetic field in the $a b$ plane would shift the spin reorientation to a higher temperature. It is worth noting that our analysis of the SRT based on MR is done in the zero-field limit.

In addition, thermal hysteresis in magnetometry was reported along with MFM images showing clear phase coexistence of the high-temperature magnetic domains with $c$ axis magnetization and low-temperature magnetic domains with in-plane magnetization [27]. The phase coexistence of out-of-plane and in-plane magnetic domains imaged by MFM support our findings of phase coexistence based on the shape of the MR curves.

A resistivity probe, which is not a direct probe of magnetization, turns out to be complimentary to the magnetometry probe and reveals information about the magnetic property of the system not readily available through magnetometry measurements. In the case of bulk magnetometry of a soft magnet, there is essentially no remnant magnetization at zero external field since magnetic domains of opposite magnetization cancel each other. To probe the magnetization using magnetometry an imbalance of up and down magnetic domains needs to be created resulting in measurements away from zero field and smearing or shifting the phase transitions. However, when probing resistivity, domains with opposite magnetization do not cancel each other, and therefore the zero-field resistivity contains information about the different magnetization directions such as $M \| \mathbf{c}$ versus $M \| \mathbf{a}$. Therefore, a careful application of magnetoresistance can be a powerful tool for probing the magnetization as we have shown in this paper.

Our finding that the electronic structure corresponding to a particular magnetization probed by AMR in the zero-field limit does not show appreciable change at the SRT despite the large redistribution of the magnetic domains is an important result that sheds light on the unexplained magnetic transition. It suggests that structural transition is probably not the drive for the SRT since a structural transition would be accompanied by a change in the electronic structure. Therefore, we believe that the cause of the SRT is more profound and relies on the nontrivial band structure of this material.
[1] E. Tang, J.-W. Mei, and X.-G. Wen, High-Temperature Fractional Quantum Hall States, Phys. Rev. Lett. 106, 236802 (2011).

[2] Z. Lin, J.-H. Choi, Q. Zhang, W. Qin, S. Yi, P. Wang, L. Li, Y. Wang, H. Zhang, Z. Sun et al., Flatbands and Emergent Ferromagnetic Ordering in $\mathrm{Fe}_{3} \mathrm{Sn}_{2}$ Kagome Lattices, Phys. Rev. Lett. 121, 096401 (2018).

[3] L. Ye, M. Kang, J. Liu, F. von Cube, C. R. Wicker, T. Suzuki, C. Jozwiak, A. Bostwick, E. Rotenberg, D. C. Bell et al., Massive Dirac fermions in a ferromagnetic kagome metal, Nature 555, 638 (2018).
[4] M. Yao, H. Lee, N. Xu, Y. Wang, J. Ma, O. V. Yazyev, Y. Xiong, M. Shi, G. Aeppli, and Y. Soh, Switchable Weyl nodes in topological kagome ferromagnet $\mathrm{Fe}_{3} \mathrm{Sn}_{2}$, arXiv: 1810.01514.

[5] J. X. Yin, S. S. Zhang, H. Li, K. Jiang, G. Chang, B. Zhang, B. Lian, C. Xiang, I. Belopolski, H. Zheng et al., Giant and anisotropic many-body spin-orbit tunability in a strongly correlated kagome magnet, Nature 562, 91 (2018).

[6] L. A. Fenner, A. A. Dee, and A. S. Wills, Noncollinearity and spin frustration in the itinerant kagome ferromagnet $\mathrm{Fe}_{3} \mathrm{Sn}_{2}$, J. Phys.: Condens Matter 21, 452202 (2009). 
[7] G. Trumpy, E. Both, C. Djéga-Mariadassou, and P. Lecocq, Mossbauer-effect studies of iron-tin alloys, Phys. Rev. B 2, 3477 (1970).

[8] G. Lecaer, B. Malaman, and B. Roques, Mossbauer-effect study of $\mathrm{Fe}_{3} \mathrm{Sn}_{2}$, J. Phys. F-Metal Phys. 8, 323 (1978).

[9] B. Malaman, D. Fruchart, and G. L. Caer, Magnetic properties of $\mathrm{Fe}_{3} \mathrm{Sn}_{2}$. II. Neutron diffraction study (and Mossbauer effect), J. Phys. F: Metal Phys. 8, 2389 (1978).

[10] M. D. Kuz'min, On the gradual character of the first-order spin reorientation transition in $\mathrm{DyFe}_{11} \mathrm{Ti}$, J. Appl. Phys. 88, 7217 (2000).

[11] A. D. Kent, J. Yu, U. Rüdiger, and S. S. P. Parkin, Domain wall resistivity in epitaxial thin film microstructures, J. Phys.: Condensed Matter 13, R461 (2001).

[12] Y. Liu, W. Chu, J. Yang, G. Liu, H. Du, W. Ning, L. Ling, W. Tong, Z. Qu, G. Cao et al., Magnetic reversal in $\mathrm{Sr}_{4} \mathrm{Ru}_{3} \mathrm{O}_{10}$ nanosheets probed by anisotropic magnetoresistance, Phys. Rev. B 98, 024425 (2018).

[13] T. Kida, L. A. Fenner, A. A. Dee, I. Terasaki, M. Hagiwara, and A. S. Wills, The giant anomalous Hall effect in the ferromagnet $\mathrm{Fe}_{3} \mathrm{Sn}_{2}$-A frustrated kagome metal, J. Phys.: Condens Matter 23, 112205 (2011).

[14] Q. Wang, S. Sun, X. Zhang, F. Pang, and H. Lei, Anomalous Hall effect in a ferromagnetic $\mathrm{Fe}_{3} \mathrm{Sn}_{2}$ single crystal with a geometrically frustrated Fe bilayer kagome lattice, Phys. Rev. B 94, 075135 (2016).

[15] B. Malaman, B. Roques, A. Courtois, and J. Protas, Crystalstructure of iron stannide $\mathrm{Fe}_{3} \mathrm{Sn}_{2}$, Acta Crystallographica Section B-Structural Science 32, 1348 (1976).

[16] G. T. Meaden, in Electrical Resistance of Metals, International Cryogenics Monograph Series, edited by K. D. T. K. Mendelssohn (Springer, Berlin, 1965).

[17] See Supplemental Material at http://link.aps.org/supplemental/ 10.1103/PhysRevB.100.214420 for further details on magnetoresistivity and fitting.

[18] B. Raquet, M. Viret, E. Sondergard, O. Cespedes, and R. Mamy, Electron-magnon scattering and magnetic resistivity in 3d ferromagnets, Phys. Rev. B 66, 024433 (2002).
[19] K. G. Rana, F. K. Dejene, N. Kumar, C. R. Rajamathi, K. Sklarek, C. Felser, and S. S. P. Parkin, Thermopower and unconventional Nernst effect in the predicted type-II Weyl semimetal $\mathrm{WTe}_{2}$, Nano Lett. 18, 6591 (2018).

[20] B. Fauqué, X. Yang, W. Tabis, M. Shen, Z. Zhu, C. Proust, Y. Fuseya, and K. Behnia, Magnetoresistance of semimetals: The case of antimony, Phys. Rev. Materials 2, 114201 (2018).

[21] Y. Feng, Y. Wang, D. M. Silevitch, J. Q. Yan, R. Kobayashi, M. Hedo, T. Nakama, Y. Ōnuki, A. V. Suslov, B. Mihaila et al., Linear magnetoresistance in the low-field limit in density-wave materials, Proc. Natl. Acad. Sci. USA 116, 11201 (2019).

[22] M. Tsunoda, Y. Komasaki, S. Kokado, S. Isogami, C.C. Chen, and M. Takahashi, Negative anisotropic magnetoresistance in $\mathrm{Fe}_{4} \mathrm{~N}$ film, Appl. Phys. Express 2, 083001 (2009).

[23] Y. You, Y. Gong, Z. Li, H. Li, M. Zhu, J. Tang, E. Liu, Y. Yao, G. Xu, F. Xu et al., Large planar topological Hall effect in a uniaxial van der Waals ferromagnet $\mathrm{Fe}_{3} \mathrm{GeTe}_{2}$, Phys. Rev. B 100, 134441 (2019).

[24] N. Eden, G. Kopnov, S. Fraenkel, M. Goldstein, and A. Gerber, Longitudinal and transverse magnetoresistance in films with tilted out-of-plane magnetic anisotropy, Phys. Rev. B 99, 064432 (2019).

[25] Z. Hou, W. Ren, B. Ding, G. Xu, Y. Wang, B. Yang, Q. Zhang, Y. Zhang, E. Liu, F. Xu et al., Observation of various and spontaneous magnetic skyrmionic bubbles at room temperature in a frustrated kagome magnet with uniaxial magnetic anisotropy, Adv. Mater. 29, 1701144 (2017).

[26] Y. Li, Q. Wang, L. DeBeer-Schmitt, Z. Guguchia, R. D. Desautels, J.-X. Yin, Q. Du, W. Ren, X. Zhao, Z. Zhang et al., Magnetic-Field Control of Topological Electronic Response near Room Temperature in Correlated Kagome Magnets, Phys. Rev. Lett. 123, 196604 (2019).

[27] K. Heritage, B. Bryant, L. A. Fenner, A. S. Wills, G. Aeppli, and Y.-A. Soh, Images of a first-order spin-reorientation phase transition in a metallic kagome ferromagnet, arXiv:1909.07768. 